\title{
Comparison of Active Contour and Active Shape Approaches for Corpus Callosum Segmentation
}

\author{
Enkhbolor Adiya ${ }^{\dagger}$, Yonny S. Izmantoko ${ }^{++}$, Heung-Kook Choi ${ }^{++\dagger}$
}

\begin{abstract}
The corpus callosum is the largest connective structure in the brain, and its shape and size are correlated to sex, age, brain growth and degeneration, handedness, musical ability, and neurological diseases. Manually segmenting the corpus callosum from brain magnetic resonance (MR) image is time consuming, error prone, and operator dependent. In this paper, two semi-automatic segmentation methods are present: the active contour model-based approach and the active shape model-based approach. We tested these methods on an MR image of the human brain and found that the active contour approach had better segmentation accuracy but was slower than the active shape approach.
\end{abstract}

Key words: Corpus callosum, distribution, segmentation, active contour, active shape

\section{INTRODUCTION}

The corpus callosum is the largest neural pathway connecting the two cerebral hemispheres of the human brain. Its nature and functions are a topic of interest in neuroimaging studies. Several medical studies have indicated that the size and shape of the corpus callosum are correlated to sex, age, brain growth and degeneration, handedness, and musical ability [1]. A variety of neurological diseases, including schizophrenia, autism, mental retardation, Down's syndrome, attention deficit

* Corresponding Author: Heung-Kook Choi, Address : (621-749) Injero 197, Gim-Hae, Gyeong-Nam, Korea, Dept. of Computer Engineering, UHRC, Inje University, Korea, TEL : +82-55-320-3437, FAX : +82-55-322-3107, E-mail : cschk@inje.ac.kr

Receipt date: May 9, 2013, Revision date: Aug. 3, 2013 Approval date: Aug. 13, 2013

${ }^{+}$Dept of Computer Engineering, Inje University, Korea (E-mail: enkhbolora@yahoo.com)

${ }^{++}$Dept of Computer Engineering, Inje University, Korea (E-mail: yonny.septian@gmail.com)

${ }^{+++}$Dept of Computer Engineering, UHRC, Inje University, Korea

(E-mail: cschk@inje.ac.kr)

※ This research was supported by Basic Science Research Program through the National Research Foundation of Korea (NRF) funded by the Ministry of Education, Science, and Technology (2012-0002646). hyperactivity disorder, Alzheimer's disease, developmental dyslexia, and developmental language disorders, are also related to the corpus callosum

The general shape of the corpus callosum generally the same in all people and its intensity in magnetic resonance (MR) images are quite different from that of its surroundings. However, it is difficult to determine its local shape. Also it remains unclear whether a part of the brain called the fornix located in the mid-sagittal region, contacts the corpus callosum because of the similar brightness of this two structures. Therefore, manual tracing is still the most common method for segmenting the corpus callosum [2-4]. However, manually segmenting the corpus callosum from brain magnetic resonance (MR) image is time consuming, error prone, and operator dependent. Therefore, it is essential to automate segmentation to some degree. In this study, we compare two image-segmentation methods: the active contour model (ACM)-based approach and the active shape model (ASM)-based approach.

The ACM was introduced by Kass et al. [5] in 1987. ACMs are among the most successful models for image segmentation, and are widely used in many applications, including those related to com- 
puter vision, pattern recognition, and medical image processing. The basic idea is to evolve a curve to segment the desired objects by minimizing energy functional subject to constraints. The existing ACMs can be categorized into boundary-based [6-8], region-based [9-12], and other higher-level prior-knowledge-based methods [13-15]. Regionbased models offer more advantages than boundary-based ones, because they are less sensitive to noise and the location of the initial contours. Accordingly, they do not use an image gradient, and provide better performance when applied to image with weak object boundaries. In regionbased segmentation, energy models employ image statistics that depend on the segmenting curve, using parametric [11,16,17] and non-parametric methods [18]. In this work, we propose maximizing the distance between two distributions to separate two regions with different probability density functions, without any prior knowledge of the object and background [19].

The ASM was proposed by Cootes et al. [20] in 1994. This method employs a statistical model derived from several sample images, and creates a mean shape model based on them. Significant eigenvalues and eigenvectors from all sample images are also utilized. In this way, the mean shape will evolve to fit the object to be segmented. ASMs are commonly used for face recognition [21,22,23] and medical image segmentation [24]. An ASM is also called a 'smart snake' [25], because it attempts to deform itself to fit the object, but only in ways that are consistent with the shapes found in the training set. Some statistical concepts and procedures, such as mean value, principal component analysis (PCA), kNN classifier, and Mahalonobis distance, are used in ASM to obtain better segmentation results.

The remainder of this paper is organized as follows. Section 2 describes the ACM and ASM approaches. In Section 3, experimental results are discussed. We state our conclusion in Section 4.

\section{Image Segmentation Methods}

\subsection{ACM approach}

\subsubsection{Description of the model}

ACMs segment objects using curve evolution starting from an initial contour. The level set method (the most suitable method for tracking moving interfaces) is implicit, parameter-free, and can easily handle topological changes. Thus we represent an evolving curve by the zero-level set of the signed distance function. We assume that our image consists of two homogeneous regions -object and background-separated by the contour. Each of these regions has a different distribution function. To segment the object and background, we need to maximize the distance between these two distributions. To measure the distance, we use a similarity metric based on prediction theory [19] and defined as follows:

$$
g_{f}(\Delta):=\int\left(\frac{\Delta}{f}\right)^{2}-\left(\int \frac{\Delta}{f}\right)^{2}
$$

This problem is equivalent to the partial differential equation (PDE) of the gradient descent method, which describes the level set evolution of an evolving curve.

Let $l \subset \mathbb{R}$, be grayscale image defined over the domain $\Omega \subset \mathbb{R}^{2},(x, y) \in \mathbb{R}^{2}$, the image coordinates, $\omega$ an open subset of $\Omega$ and $C=\partial \omega$ its boundary. In the level set method [26], the curve $C$ is represented by the zero-level set of the signed distance function $\phi: \Omega \rightarrow \mathbb{R}$, such that

$$
\left\{\begin{array}{l}
C=\partial \omega=\{(x, y) \in \Omega: \phi(x, y)=0\} \\
\text { Inside }(C)=\{(x, y) \in \Omega: \phi(x, y)<0\} \\
\text { Outside }(C)=\{(x, y) \in \Omega: \phi(x, y)>0\}
\end{array}\right.
$$

In Fig. 1, we illustrate the above notations for level set function $\phi$, used to represent the curve C.

Let $p^{-}(z, \phi)$ and $p+(z, \phi)$ be the respective probability density functions of the inside and outside regions of curve $C$. Using the Heaviside func- 


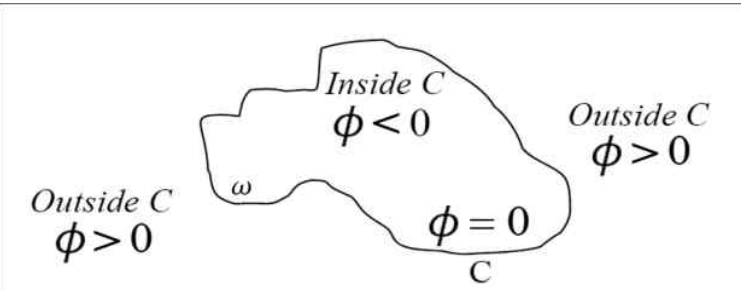

$\Omega$

Fig. 1 Level set representation of curve C

tion (1) we can write the cumulative density function of the inside region of the curve $C$ as (2). $p^{-}(z$, $\phi)$ is defined by (3), where $A \_(\phi(x, y))$ is the area of the inside region of curve $C$ and $\delta(\phi)$, the Dirac delta function, is the derivative of (1). In a similar way, we obtain (3) and define the probability density function $p+(Z, \phi)$ by (4).

$$
\begin{aligned}
\theta(\phi)=\left\{\begin{array}{l}
1, \phi \geq 0 \\
0, \phi<0
\end{array}\right. & \begin{aligned}
F_{-}(z, \phi) & =\frac{\int_{\text {inside }(C)} \theta(z-I(x, y)) d x d y}{\int_{\text {inside }(C)} d x d y} \\
& =\frac{\int_{\Omega} \theta(z-I(x, y)) \theta(-\phi(x, y)) d x d y}{\int_{\Omega} \theta(-\phi(x, y)) d x d y} \\
& =\frac{\int_{\Omega} \theta(z-I(x, y)) \theta(-\phi(x, y)) d x d y}{A_{-}(\phi(x, y))} \\
p_{-}(z, \phi) & =\frac{d F_{-}(z, \phi)}{d z} \\
& =\frac{\int_{\Omega} \delta(z-I(x, y)) \theta(-\phi(x, y)) d x d y}{A_{-}(\phi(x, y))} \\
p_{+}(z, \phi) & =\frac{\int_{\Omega} \delta(z-I(x, y)) \theta(\phi(x, y)) d x d y}{A_{+}(\phi(x, y))}
\end{aligned}
\end{aligned}
$$

The log-likelihood ratio (5) of the two distributions is used to measure the dissimilarity of the distributions. In ACM, we maximize the fitting term (6) as the standard deviation of the log-likelihood ratio function.

$$
\begin{aligned}
& \mathrm{L}(\mathrm{z}, \phi)=\log \frac{p_{-}(z, \phi)}{p_{+}(z, \phi)} \\
& E_{\text {image }}(z, \phi)=\sqrt{\mathcal{E}\left\{L(z, \phi)^{2}\right\}-\mathcal{E}\{L(z, \phi)\}^{2}}
\end{aligned}
$$

$\varepsilon\{f(z)\}$ is the expected value of the function $f(z)$, where $z$ is a random variable in the range [0,255].

In ACM, a regularizing term is added to the fitting term (6), and hence the energy functional minimization problem is defined by (7). Here, $\lambda \geq 0$ is a weight parameter, and minimization of the length of the curve, length $(\phi=0)$, is performed under the assumptions of regularization and a smooth contour. Applying the gradient descent method to this optimization problem, we obtain the PDE (8), which describes the level set evolution of the curve $C$ that maximizes the distance between the distributions of the inside and outside regions of the segmenting curve. We compute the gradient of the fitting term (6) using equation (9).

$$
\begin{aligned}
& -E_{\text {image }}(z, \phi)+\lambda \cdot \text { length }(\phi=0) \rightarrow \min \\
& \frac{\partial \phi}{\partial t}=\nabla_{\phi} E_{\text {image }}+\lambda \delta(\phi) \operatorname{div}\left(\frac{\nabla(\phi)}{|\nabla(\phi)|}\right) \\
& \nabla_{\phi} E_{\text {image }}=\frac{\delta(\phi)}{E_{\text {image }}} \cdot[\mathcal{E}\{L \cdot G\}-\mathcal{E}\{L\} \cdot \mathcal{E}\{G\}]
\end{aligned}
$$

where,

$$
\begin{aligned}
G= & {\left[\left(\frac{1}{A_{-}(\phi)}+\frac{1}{A_{+}(\phi)}\right)-\delta(z-I(x, y))\right.} \\
& \left.\left(\frac{1}{A_{-}(\phi) p_{-}(z, \phi)}+\frac{1}{A_{+}(\phi) p_{+}(z, \phi)}\right)\right]
\end{aligned}
$$

\subsubsection{Numerical approximation of the model}

For the numerical experiments on level set evolution we used the regularizations of the Heaviside function and Dirac delta function given by (10) and (11), respectively. These regularizations are shown in Fig. 2.

$$
\begin{aligned}
& \theta_{\varepsilon}(\phi)=\left\{\begin{array}{l}
1, \phi>\varepsilon \\
0, \phi<-\varepsilon \\
\frac{1}{2}\left(1+\frac{\phi}{\varepsilon}+\frac{1}{\pi} \sin \left(\frac{\pi \phi}{\varepsilon}\right)\right),-\varepsilon \leq \phi \leq \varepsilon
\end{array}\right. \\
& \delta_{\varepsilon}(\phi)=\left\{\begin{array}{l}
0, \phi>\varepsilon, \phi<-\varepsilon \\
\frac{1}{2 \varepsilon}\left(1+\cos \left(\frac{\pi \phi}{\varepsilon}\right)\right),-\varepsilon \leq \phi \leq \varepsilon
\end{array}\right.
\end{aligned}
$$

To discretize equation (8) with respect to $\phi$, we used a finite difference scheme; and the following notation. Let $\Delta t$ be the time step, and $\left(x_{i}, y_{j}\right)=(i$, 


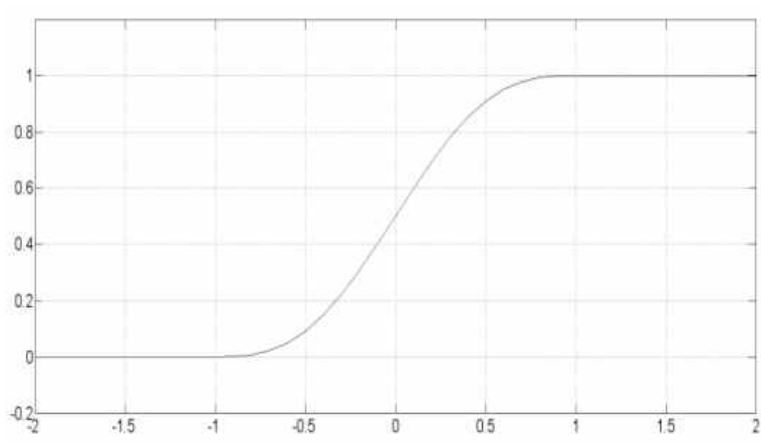

(a)

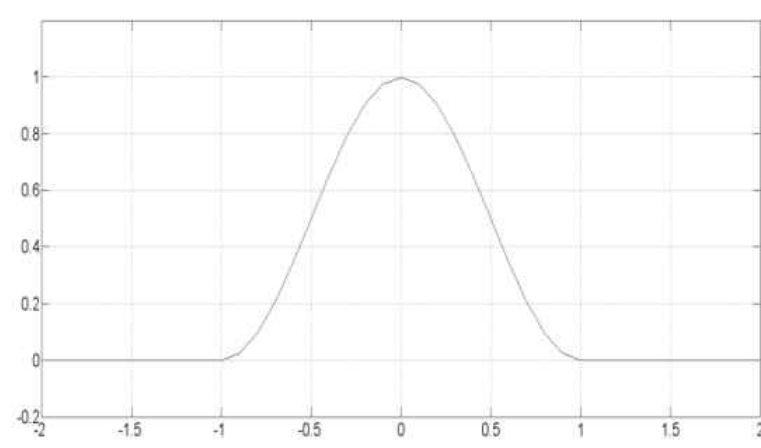

(b)

Fig. 2 The regularizations of the (a) Heaviside function and (b) Dirac delta function

$j)$ be the image pixels, for $i=1, \cdots, M, j=1, \cdots N$. Let $\phi_{i, j}^{n}=\left(n \Delta t, x_{i}, y_{j}\right)$ be an approximation of $\phi(t, x, y$, with $n \geq 0, \phi^{0}=\phi_{0}$, here $\phi_{0}$ is the initial contour. The finite differences are

$$
\begin{aligned}
\Delta_{-}^{x} \phi_{i, j}= & \phi_{i, j}-\phi_{i-1, j}, \\
& \Delta_{+}^{x} \phi_{i, j}=\phi_{i+1, j}-\phi_{i, j} \\
\Delta_{-}^{y} \phi_{i, j}= & \phi_{i, j}-\phi_{i, j-1}, \\
& \Delta_{+}^{y} \phi_{i, j}=\phi_{i, j+1}-\phi_{i, j}
\end{aligned}
$$

Then, we compute $\phi^{n+1}$ via the following discretization and linearization of (12) with respect to $\phi$.

$$
\begin{aligned}
& \frac{\phi_{i, j}^{n+1}-\phi_{i, j}^{n}}{\Delta t}=\nabla_{\phi} E_{\text {image }}\left(\phi_{i, j}^{n}\right)+ \\
& \lambda \delta_{\varepsilon}\left(\phi_{i, j}^{n}\right)\left[\Delta_{-}^{x}\left(\frac{\Delta_{+}^{x} \phi_{i, j}^{n}}{\sqrt{\left(\Delta_{+}^{x} \phi_{i, j}^{n}\right)^{2}+\left(\left(\phi_{i, j+1}^{n}-\phi_{i, j-1}^{n}\right) / 2\right)^{2}}}\right)\right. \\
&\left.+\Delta_{-}^{y}\left(\frac{\Delta_{+}^{y} \phi_{i, j}^{n}}{\sqrt{\left(\Delta_{+}^{y} \phi_{i, j}^{n}\right)^{2}+\left(\left(\phi_{i, j+1}^{n}-\phi_{i, j-1}^{n}\right) / 2\right)^{2}}}\right)\right]
\end{aligned}
$$

The principal steps of the algorithm are as follows:

- Initialize $\phi^{0}$ by $\phi_{0}, n=0$.

- Repeat

○ Compute $p-(z, \phi), A-(\phi)$ and $p+(z, \phi), A+$ $(\phi)$, using (3) and (4), respectively.

○ Solve the PDE in $\phi$ via (12), to obtain $\phi^{n+1}$.

$\circ$ Reinitialize $\phi$.

- Until the algorithm converges.

\subsection{ASM approach}

ASMs use prior knowledge obtained from a number of sample images. In ASM, we use data from each sample image to construct a model that can be used to segment another image. There are four main steps in the procedure: training, modeling, initialization and segmentation.

- In the training step, landmark points are placed on the sample images to create the training set. This process must be carried out manually for each image to obtain the boundary of the object to be segmented.

- The modeling step employs all of the data $\mathrm{ac}^{-}$ quired in the training step. The landmark points from each image must be aligned before constructing the model. The model consists of a shape model (created from the mean positions of all landmark points in all sample images) and a gray level appearance model (created using the gray level intensity along the normal at each landmark point).

- Initialization is an important step in ASM. Incorrect placement of the model on the object can result in poor segmentation. Therefore, the model should be placed as close as possible to the object.

- In the segmentation step, each landmark point of the model evolves to fit the contour of the object. 


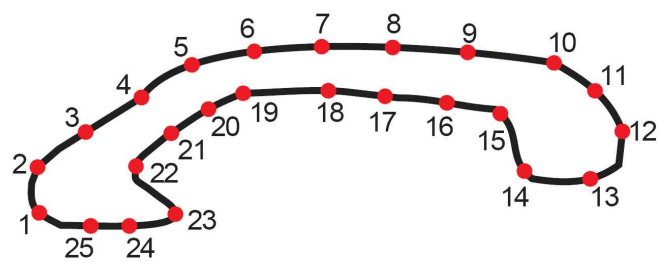

Fig. 3 The order of placement of landmark points on the corpus callosum.

\subsubsection{Training}

The goal of this step is to create a training set in which the shape of the corpus callosum is $\mathrm{ex}^{-}$ pressed by the coordinates of the landmark points. It begins with manual placement of landmark points on each sample image, and is therefore the most time ${ }^{-}$consuming step in ASM. Naturally, more landmark points will result in a more accurate shape. In our example, we chose to put 25 landmark points on the corpus callosum. The order of these landmark points is shown in Fig. 3. This is accomplished by putting landmark points on the corners of the corpus callosum and inserting some points between the corners.

We would like to obtain an accurate representation of the shape of the corpus callosum, but 25 landmark points are not enough to accomplish this. Therefore, we automatically insert more landmark points between our original landmark points using interpolation. The interpolation procedure can be considered semi-automatic landmarking because we must first manually provide the original 25 points. The goal of this interpolation process is not only to obtain a more accurate representation of the shape of the corpus callosum, but also to provide a simple way of examining the effect of the number of landmark points in ASM.

The shape of the corpus callosum is represented by a set of $n$ points. These points are represented by the vector $x_{i}$ in (13), where $n$ is the number of landmark points and $i$ is the index of the sample image.

$$
\boldsymbol{x}_{\boldsymbol{i}}=\left(x_{i 1}, y_{i 1} ; x_{i 2}, y_{i 2} ; \ldots ; x_{i n}, y_{i n}\right)^{T}
$$

\subsubsection{Modeling}

ASM examines the statistics of the coordinates of the labeled points in the training set. The various positions of the corpus callosum in the different sample images will lead to different landmark point coordinates. Thus it is necessary to align all shapes acquired in the learning step before performing any statistical computations. There are several shapealignment methods; one of which is generalized procrustes analysis (GPA) which is used to align a set of objects [27].

In GPA, transformations, such as translation, scaling, and rotation, are applied to the original $\mathrm{po}^{-}$ sition of the shape. In our work, we move every shape to the base of the coordinates before applying any transformation. This is done by updating the value of each coordinate in a shape by adding the mean value of all the corresponding coordinates in that shape (translation offset).

Rotational variation can be removed by using the direction of each point in a shape. We compute the mean direction of the shape (rotation offset), and use it to update the direction of each point. The last step of this alignment is to create new points with updated directions, which will be used for further statistical computations. Fig. 4 shows an out-

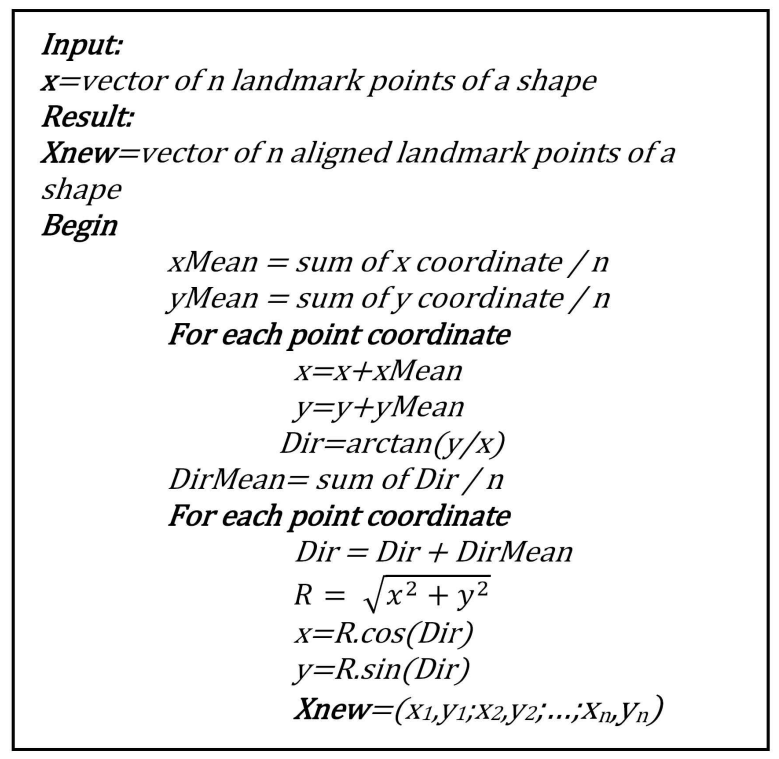

Fig. 4 The shape alignment algorithm. 
line of the alignment process.

After aligning all shapes in the training set, we compute the mean shape of the training set using (14), where $x_{i}$ is the vector of all coordinates in a shape, and $\mathrm{m}$ is the number of shapes used in the training set.

$$
\overline{\boldsymbol{x}}=\frac{1}{m} \sum_{i=1}^{m} \boldsymbol{x}_{\boldsymbol{i}}
$$

Fig. 5 shows the mean shape of the corpus callosum acquired from the training set. Thus the mean shape will be the base of all shape varieties that will be used in ASM. The variation in shape results from the movement of the landmark points based on eigenvalues and eigenvectors. The $\mathrm{CO}^{-}$ variance of all of the training data is computed using (15). From this covariance, we can obtain eigenvalues $\lambda_{i}$ and eigenvectors $s p_{i}$. To limit the number of eigenvalues, we only use significant $e^{-}$ genvalues that are higher than $98 \%$ of all of the eigenvalues.

$$
\mathrm{S}=\frac{1}{m-1} \sum_{i=1}^{m}\left(\boldsymbol{x}_{\boldsymbol{i}}-\overline{\boldsymbol{x}}\right)\left(\boldsymbol{x}_{\boldsymbol{i}}-\overline{\boldsymbol{x}}\right)^{T}
$$

The model can be approximated with (16) using the mean shape, significant eigenvectors, and a weight vector $b$. The value of $b$ is determined by (17). It is usually bounded by a multiple of the square root of an eigenvalue $\lambda_{i}$ as in (18). The variation in the mean shape caused by some of the eigenvalues is shown in Table 1 . The first significant eigenvalue adjusts the thickness and size of the corpus callosum. The second eigenvalue changes the position of the left part of the corpus callosum. The third and fifth eigenvalues adjust the respective curvatures of the lower and upper parts

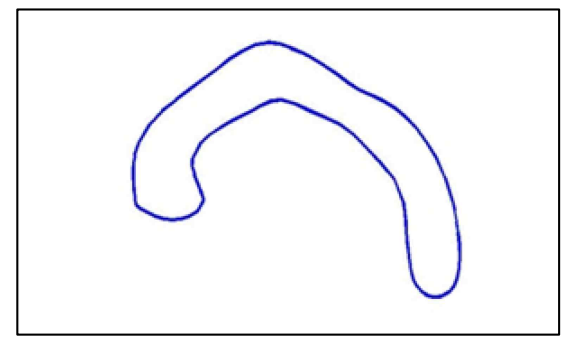

Fig. 5 The mean shape of the corpus callosum
Table 1. Mean shape variation in the corpus

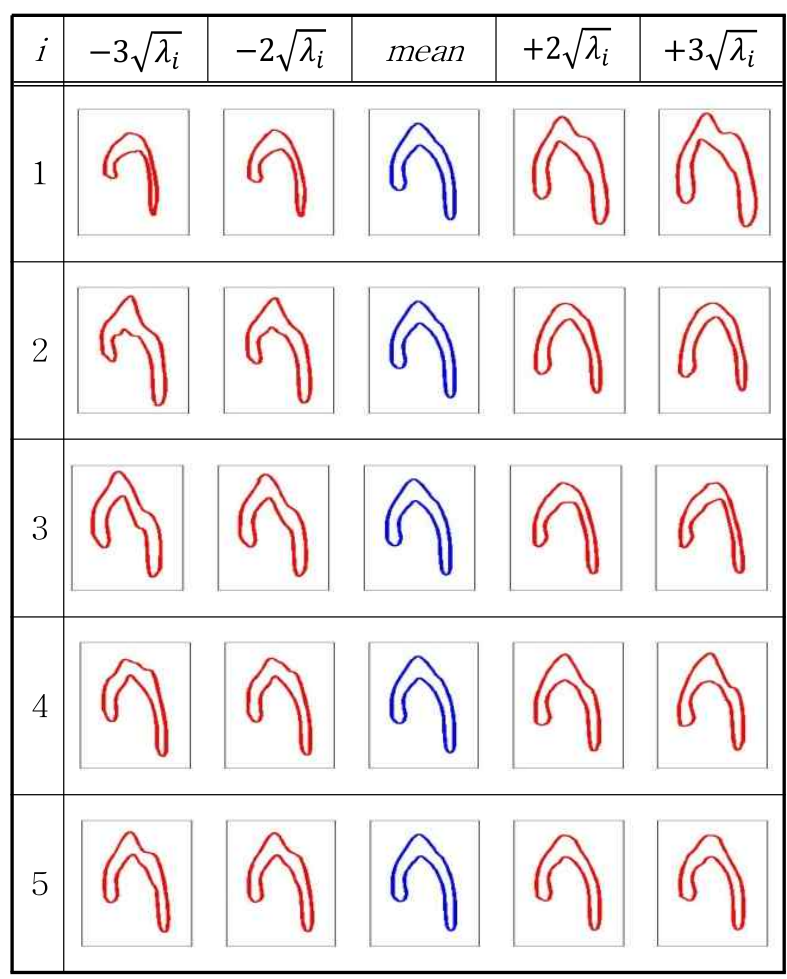

of the corpus callosum, whereas the fourth $\mathrm{ei}^{-}$ genvalue adjusts the top left part.

$$
\begin{aligned}
& \mathbf{x}=\overline{\boldsymbol{x}}+P \boldsymbol{b} \\
& \mathbf{b}=P^{T}(\boldsymbol{x}-\overline{\boldsymbol{x}}) \\
& -3 \sqrt{\lambda_{i}} \leq b_{i} \leq 3 \sqrt{\lambda_{i}}
\end{aligned}
$$

The next step is to create a gray level appearance. Its function is to obtain the mean gray level profile along the normal at each landmark point. We choose $\mathrm{k}$ points outside the landmark point and $\mathrm{k}$ points inside the landmark point, so that we have $2 \mathrm{k}^{+1}$ points in the gray level profile. We create the mean gray level profile for all shapes in the training set.

\subsubsection{Initialization}

This is the process that places the mean shape on the object to be segmented. It is important to place the mean shape as precisely as possible. Misplacing the mean shape may result in incorrect segmentation. 


\subsubsection{Segmentation}

Segmentation is the last step in ASM. It utilizes the shape model and gray profile obtained in the modeling step and the position of the mean shape from the initialization step. The main thing that happens in segmentation is the evolution of the mean shape, which is determined by the movement of each landmark point. The distance of point movement along the normal is defines the gray level resolution [28]. This resolution can vary from coarse (a long distance between each pair of profile points) to fine (a short distance between each pair of profile points). Thus, each point moves along its normal by minimizing the Mahalanobis distance between the mean gray profile, $\bar{g}$, and the gray profile of the image, $g_{i}$ given by (19) [29].

$$
\mathrm{f}\left(g_{i}\right)=\left(g_{i}-\bar{g}\right) S_{g}^{-1}\left(g_{i}-\bar{g}\right)
$$

The segmentation is expected to converge. However, the number of iterations must still be adequate to ensure that the stop condition of the procedure is satisfied.

\section{EXPERIMENTAL RESULTS}

The test image was acquired using a Siemens
3T MR imaging device from Pusan National University Hospital, Pusan, South Korea. The subjects were three normal males, 24-27 years of age. The images were a T1-weighted grayscale DICOM image with a resolution $256 \times 256$. There are various parameters that can influence the segmentation results. The initializations for both methods are simple and can be done by any user. In ACM case, user needs to draw any contour inside the object, and in ASM case, user needs to put the mean shape as close as possible to the target object. Fig. 6(a) shows the original image I, Fig. 6(b) shows the initial contour and Fig. 6(b-d) shows the segmentation results of ACM. Fig. 6(e, f) shows the initialization and respective ASM results with 2 and 20 interpolated points.

The ground truth image is shown in Fig. 7(a), which is considered the benchmark for the corpus callosum area and the segmentation results of $\mathrm{ACM}$ and ASM are shown in Fig. 7(b) and Fig. 7(c), respectively.

To evaluate the corpus callosum segmentation accuracy as a segmentation error in percentage, we used Jaccard distance, $J_{\delta}(A, B)$, as follows:

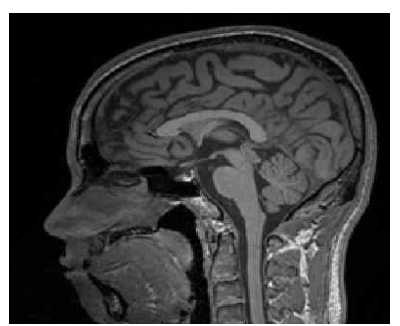

(a) Original image I

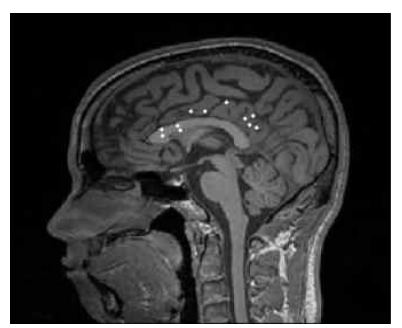

(e) 2 interpolated points: Initial and final conditions

(b) Initial contour
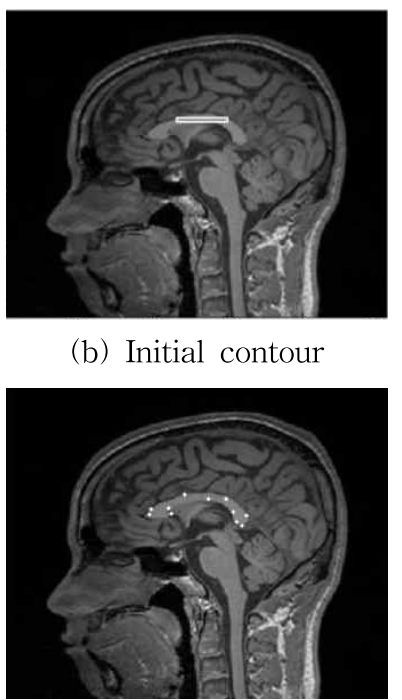
Fig. 6. First row: Initial contour and ACM segmentation results; Second row: initial position of mean
shapes and ASM segmentation results;

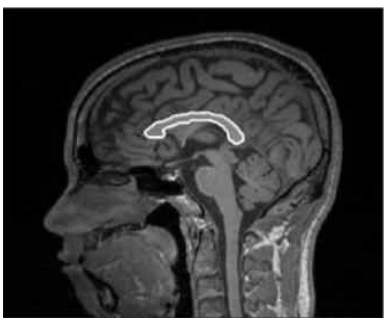

(c) 1100 iterations

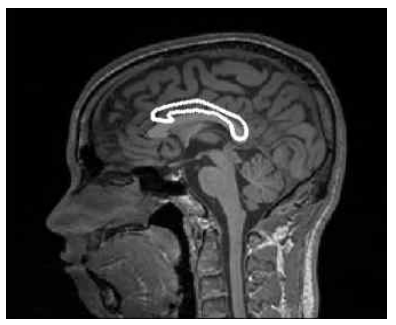

(f) 20 interpolated points: Initial and final conditions

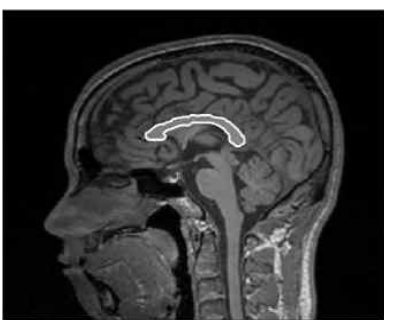

(d) 1400 iterations

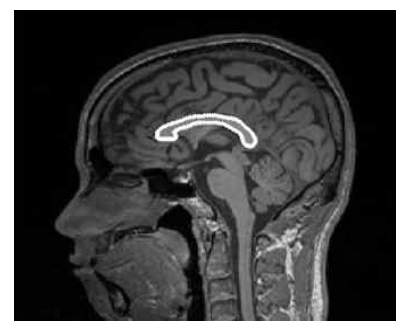




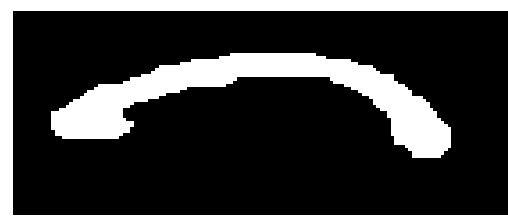

(a) Ground truth image

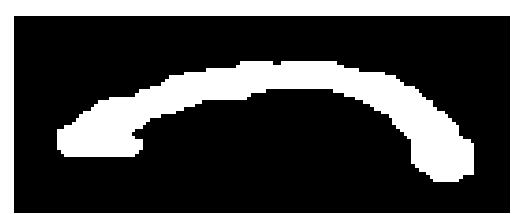

(b) ACM segmentation result

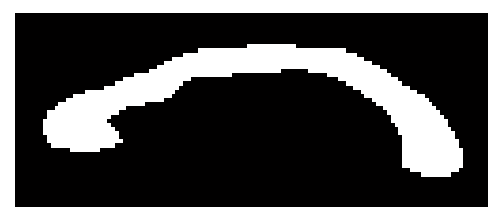

(c) ASM segmentation result

Fig. 7 (a) Ground truth image; The area of the corpus callosum using (b) ACM, (c) ASM;

$$
\begin{aligned}
\text { Segmentation error } & =J_{\delta}(A, B) \cdot 100 \% \\
& =(1-J(A, B)) \cdot 100 \%
\end{aligned}
$$

where $A$ is the ground truth image, $B$ is the segmentation result and Jaccard similarity coefficient (also known as Jaccard index) defined as

$$
J(A, B)=\frac{|A \cap B|}{|A \cup B|}
$$

In ACM, the segmentation result depends on the initial contour and the number of iterations. Fig. 8 shows that the elapsed time (gray) increased with the number of iterations, while the percentage error (black) first decreased and then became stable. The segmentation time and accuracy for numbers of iterations between 100 and 2000 are listed in Table 2. The error was stable after 1200 iterations. This was our optimum result for ACM.

In ASM, we varied the number of landmark points. Point-to-point interpolation was used to include more landmark points in the model. Among the original 25 landmark points of the corpus cal- losum model, there were 11 corner points which were the starting and end points of the interpolation. There were actually only 10 such points because the starting and end points were the same. Therefore, each interpolation produced a total number of landmark points determined by (20).

\section{Total landmark points}

$$
=11 \times \text { interpolated points }-10
$$

As mentioned before, the initialization step is important in ASM. The more precise the placement of the model on the image, the better the segmentation will be. However, we used the same initial position in all experimental tests, because we wanted comparable results. Fig. 9 shows that the percentage error (black line) clearly decreased when the total number of landmark points was between 10 and 45. Beyond 45, the percentage error remained at roughly the same level, around 19-21 $\%$. On the other hand, the elapsed time (gray line) increased with the number of landmark points. The

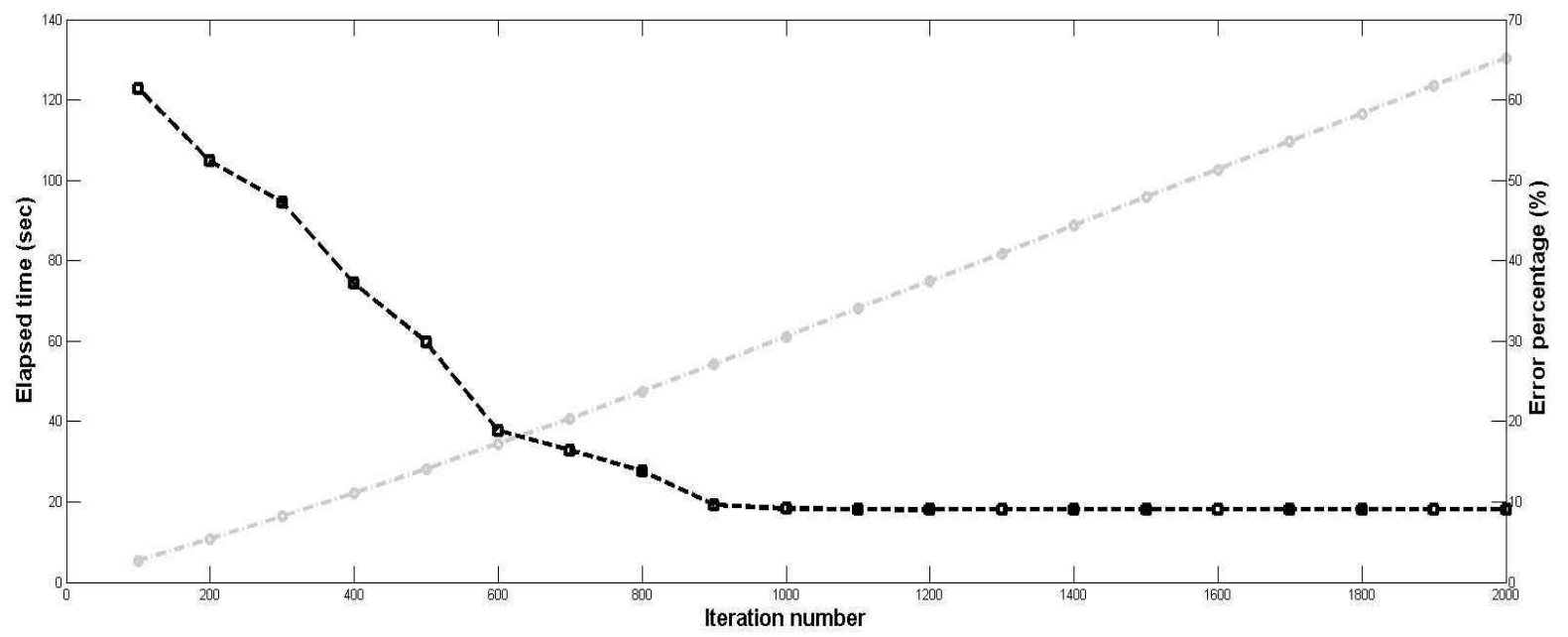

Fig. 8. Graphs of the number of iterations vs. percentage error (black) and the number of iterations vs. elapsed time (gray). 
Table

2. Elapsed Time and segmentation error
using ACM

\begin{tabular}{|c|c|c|}
\hline Iteration numbers & Time elapsed $(\mathrm{s})$ & Error $(\%)$ \\
\hline \hline 100 & 5.32 & 61.38 \\
200 & 10.71 & 52.37 \\
300 & 16.33 & 47.24 \\
400 & 22.05 & 37.15 \\
500 & 28.12 & 29.89 \\
600 & 34.36 & 18.85 \\
700 & 40.74 & 16.39 \\
800 & 47.47 & 13.81 \\
900 & 54.29 & 9.65 \\
1000 & 61.14 & 9.12 \\
1100 & 68.02 & 9.06 \\
1200 & 74.88 & 8.99 \\
1300 & 81.81 & 8.99 \\
1400 & 88.79 & 8.99 \\
1500 & 95.77 & 8.99 \\
1600 & 102.70 & 8.99 \\
1700 & 109.61 & 8.99 \\
1800 & 116.58 & 8.99 \\
1900 & 123.47 & 8.99 \\
2000 & 130.30 & 8.99 \\
\hline
\end{tabular}

details are listed in Table 3.

This fluctuation in percentage error was caused by the model shape. When the number of interpolated points was 2 or 3 , the corpus callosum model was not clear or smooth, and the segmentation result had a high percentage error. When the number of landmark points was 45 (interpolated by 5 points), the shape of the corpus callosum model
Table 3. Influence of the number of points interpolated between landmarks

\begin{tabular}{|c|c|c|c|}
\hline $\begin{array}{c}\text { Interpolated } \\
\text { points }\end{array}$ & $\begin{array}{c}\text { Total number of } \\
\text { landmark points }\end{array}$ & $\begin{array}{c}\text { Time } \\
\text { elapsed }(\mathrm{s})\end{array}$ & $\begin{array}{c}\text { Error } \\
(\%)\end{array}$ \\
\hline \hline 2 & 12 & 6.48 & 41.46 \\
3 & 23 & 6.87 & 26.27 \\
5 & 45 & 7.53 & 21.08 \\
10 & 100 & 9.84 & 19.79 \\
15 & 155 & 12.07 & 19.72 \\
20 & 210 & 14.59 & 19.47 \\
25 & 265 & 16.71 & 19.83 \\
30 & 320 & 18.54 & 21.24 \\
35 & 375 & 19.96 & 20.57 \\
40 & 430 & 22.47 & 20.00 \\
45 & 485 & 25.24 & 20.20 \\
50 & 540 & 27.09 & 21.39 \\
\hline
\end{tabular}

was already formed. Fig. 10 shows the differences between the shapes of the corpus callosum models for 3, 5, 25, and 50 interpolations. There were no major differences between the shapes produced by 5,25 , and 50 interpolations. A good corpus callosum shape was obtained with only 5 interpolated points. More interpolated points merely resulted in a shorter distance between points, without any significant shape change. Therefore, the segmentation results were virtually the same for all cases from 5 to 50 interpolated points, with little variation. This variation was caused by using the same initial position for all models, because one model is unlikely to have the same optimal initial position as

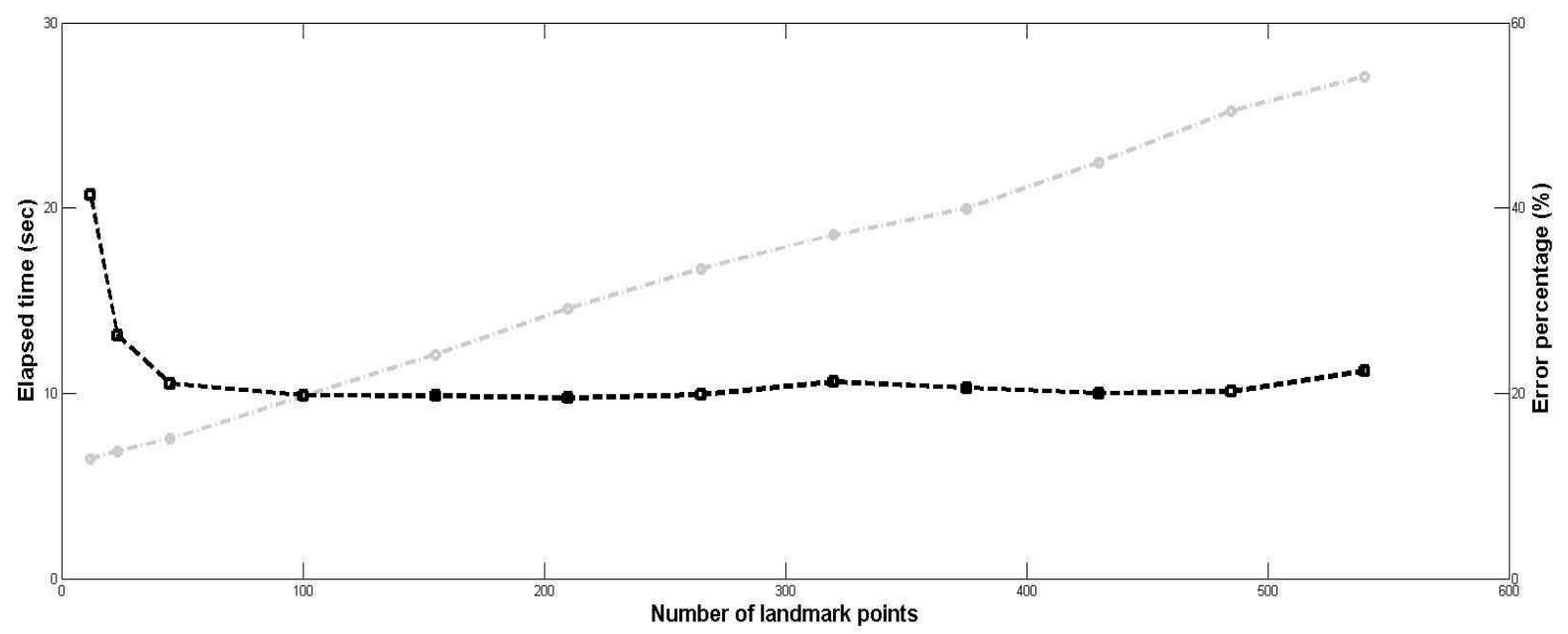

Fig. 9. Graphs of landmark points vs. percentage error (black) and landmark points vs. elapsed time (gray). 


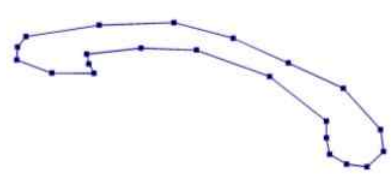

(a)

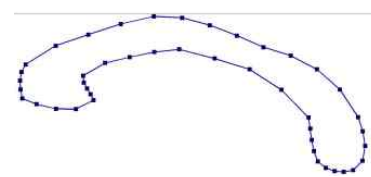

(b)

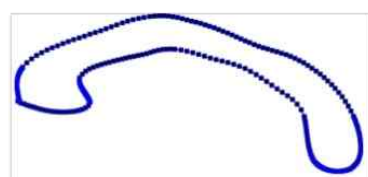

(c)

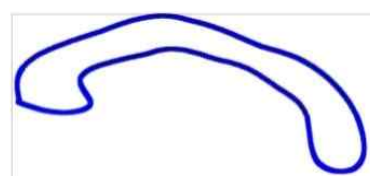

(d)

Fig. 10. Corpus callosum models for some interpolated points: (a) 3, (b) 5, (c) 25, and (d) 50 points.

another. In our experiments, 20 interpolated points produced the optimum ASM corpus callosum segmentation.

In Fig. 11, first row shows original image II, its respective segmentation results of $\mathrm{ACM}$ and ASM, and second row shows original image III, its segmentation results of ACM and ASM, respectively. Comparison of segmentation accuracy and elapsed time of ACM and ASM is shown in Table 4.

\section{CONCLUSION}

In our experiments, it was clearly shown that
ASM is faster than ACM. To obtain the best results, ASM required around 10-16 seconds, while ACM required around 60-81 seconds. This means that ASM is more than 5 times faster than ACM. On the other hand, the segmentation accuracy of ACM was much better than that of ASM. The ACM segmentation error was 8.61-12.72\%, whereas, the ASM error was 19.07-26.99\%. These results indicate a tradeoff between the segmentation accuracy of ACM and the segmentation time of ASM. However, in medical image segmentation, accuracy is more important than time and it must be the main consideration in choosing a segmenta-

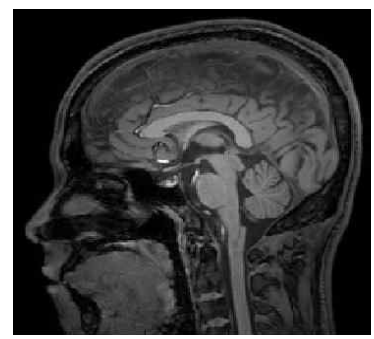

(a) Original image II

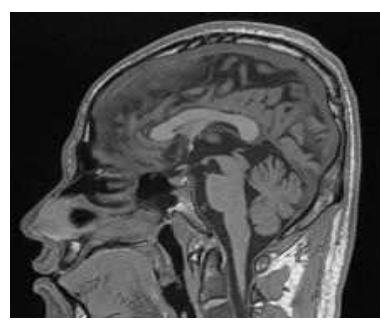

(d) Original image III

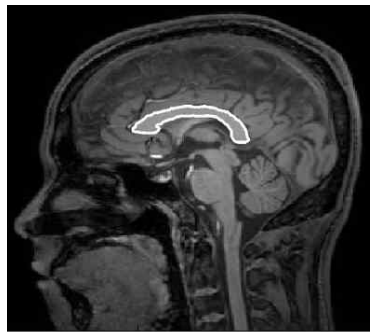

(b) ACM segmentation result

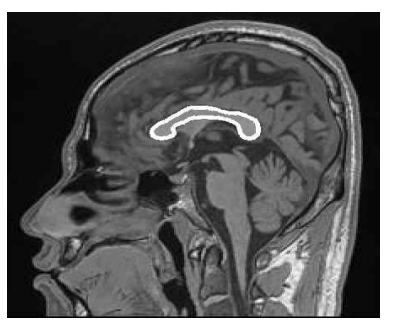

(e) ACM segmentation result

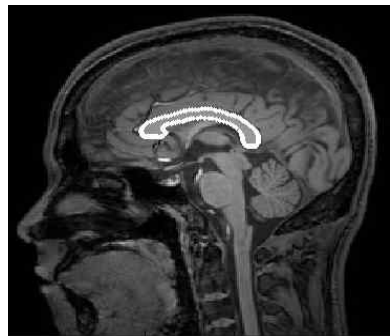

(c) ASM segmentation result

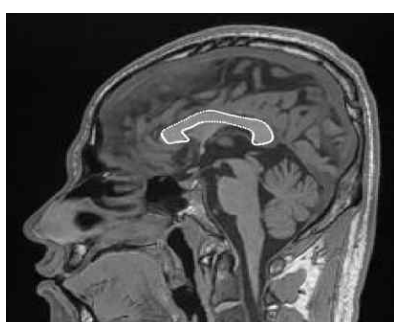

(f) ASM segmentation result

Fig. 11. First column: Original images; Second and third column: The segmentation results of ACM and ASM, respectively;

Table 4. Comparison of the ACM and ASM

\begin{tabular}{|c|c|c|c|c|}
\hline \multirow{2}{*}{} & \multicolumn{2}{|c|}{ Elapsed time $(\mathrm{sec})$} & \multicolumn{2}{c|}{ Error (\%) } \\
\cline { 2 - 5 } & ACM & ASM & ACM & 19.47 \\
\hline \hline Original image I & 74.88 & 14.59 & 8.99 & 26.99 \\
\hline Original image II & 81.46 & 16.21 & 12.72 & 19.07 \\
\hline Original image III & 59.70 & 10.47 & 8.61 & \\
\hline
\end{tabular}


tion method, because error could lead to an erroneous diagnosis of a patient's medical status or disease. Therefore, to segment the corpus callosum, ACM is preferable to ASM.

\section{REFERENCES}

[1] A. Elsayed, F. Coenen, M.G. Finana, and V. Sluming, "MRI Brain ScCn Classification According to the Nature of the Corpus Callosum," Syposium on Med. Image Underst. and Appl., pp. 19-23, 2010.

[2] C.N. Vidal, R. Nicolson, T.J. DeVito, K.M. Hayashi, J.A. Geaga, D.J. Drost, P.C. Williamson, N. Rajakumar, Y. Sui, R. A. Dutton, A.W. Toga and P.M. Thompson, "Mapping Corpus Callosum Deficits in Autism: An Index of Aberrant Cortical Connectivity," Biological Psychiatry, Vol. 60, No. 3, pp. 218225, 2006.

[3] J. Piven, J. Bailey, B.J. Ranson, and S. Arndt, "An MRI Study of the Corpus Callosum in Autism," Am. J. of Psychiatry, Vol. 154, No. 8, pp. 1051-1056, 1997.

[4] S.L. Palmer, W.E. Reddick, J.O. Glass, A. Gajjar, O. Goloubeva, and R.K. Mulhern, "Decline in Corpus Callosum Volume Among Pediatric Patients with Medulloblastoma: Longitudinal MR Imaging Study," Am. J. of Neuroradiol., Vol. 23, No. 7, pp. 1088-1094, 2002.

[5] A. Kass and D. Terzopoulos, "Snakes: Active Contour Models," Int. J. of Comput. Vis., Vol. 1, No. 4, pp. 321-331, 1987.

[6] L. Cohen, "On Active Contour Models and Balloons," Comput. Vis., Graphics and Image Process. Image Underst., Vol. 53, No. 2, pp. 211-218, 1991.

[7] V. Caselles, F. Catte, T. Coll, and F. Dibos, "A Geometric Model for Active Contours in Image Processing," Numerische Mathematik, Vol. 66, No. 1, pp. 1-31, 1993.
[8] V. Caselles, R. Kimmel, and G. Sapiro, "Geodesic Active Contours," Int. J. of Comput. Vis., Vol. 22, No. 1, pp. 61-79, 1997.

[9] S.C. Zhu and A. Yuille, "Region Competition: Unifying Snakes, Region Ggrowing, and Bayes/MDL for Multiband Image Segmentation," IEEE Trans. on Pattern Anal. and Machine Intell., Vol. 18, No. 9, pp. 884-900, 1996.

[10] D. Mumford and J. Shah, "Optimal Approximations by Piecewise Smooth Functions and Associated Variational Problems," Commun. on Pure and Appl. Math., Vol. 42, No. 5, pp. 577-685, 1989.

[11] T. Chan and L. Vese, "Active Contours Without Edges," IEEE Trans. on Image Process., Vol. 10, No. 2, pp. 266-277, 2001.

[12] J. Mille, "Narrow Band Region-based Active Contours and Surfaces for 2D and 3D Segmentation," Comput. Vis. and Image Underst., Vol. 113, No. 9, pp. 946-965, 2009.

[13] S. Dambreville, Y. Rathi, and A. Tannenbaum, "Shape-based Approach to Robust Image Segmentation using Kernel PCA," IEEE Conf on Comput. Vis, and Pattern Recognit., Vol. 1, pp. 977-984, 2006.

[14] Y. Chen, F. Huang, and H. Tagare, "Using Prior Shapes in Geometric Active Contours in a Variational Framework," Int. J. of Comput. Vis., Vol. 50, No.3, pp. 315-328, 2007.

[15] M. Leventon, E. Grimson, and O. Faugeras, "Statistical Shape Influence in Geodesic Active Contours," IEEE Conf. on Comput. Vis. and Pattern Recognit., pp. 1316-1324, 2000.

[16] M. Rousson and R. Deriche, "A Variational Framework for Active and Adaptive Segmentation of Vector Valued Images," IEEE Workshop on Motion and Video Comput., pp. 56-62, 2002.

[17] A. Yezzi, A. Tsai, and A. Willsky, "A Statistical Approach to Snakes for Biomodal Trimodal Imagery," Int. Conf. on Comput. Vis., pp. 898- 
903, 1999.

[18] J. Kim, J. Fisher, A. Yezzi, M. Cetin, and A. Willsky, "A Nonparametric Statistical Method for Image Segmentation using Information Theory and Curve Evolution," IEEE Trans. on Image Process., Vol. 14, No. 10, pp. 14861502, 2005.

[19] R. Sandhu, T. Georgiou, and A. Tannenbaum, "A New Distribution Metric for Image Segmentation," SPIE Med. Imaging, Vol. 6914, pp. 691404-1-9, 2008.

[20] T.F. Cootes, C.J. Taylor, D.H. Cooper, and J. Graham, "Active Shape Models - Their Training and Application," Comput. Vis. and Image Underst., Vol. 61, No. 1, pp. 38-59, 1995.

[21] C. Butakoff and A.F. Frangi, "Multi-view Face Segmentation using Fusion of Statistical Shape and Appearance Models," Comput. Vis. and Image Underst., Vol. 114, No. 3, pp. 311321, 2010.

[22] D.A. Lim and J.P. Ko, " A System for Recognizing Sunglasses and a Mask of an ATM user," Journal of Korea Multimedia Society, Vol. 11, No. 1, pp. 34-43, 2008

[23] W.C. Song, S.K. Kang, and T.S. Jung, "An Automatic Smile Analysis System for Smile self-training," Journal of Korea Multimedia Society, Vol. 14, No. 11, pp. 1373-1382, 2011.
[24] T.F. Cootes, A.J. Hill, C.J. Taylor, and J. Haslam, "The use of Active Shape Models for Locating Structures in Medical Images," Image and Vision Comput., Vol. 12, No. 6, pp. 355-366, 1994.

[25] T.F. Cootes and C.J. Taylor, "Active Shape Models-Smart Snakes," Proc. of the Br. Machine Vis. Conf, pp. 266-275, 1992.

[26] S. Osher and J.A. Sethian, "Fronts Propagating with Curvature-dependent Speed: Algorithms Based on Hamilton-Jacobi Formulation," J. of Comput. Phys., Vol. 79, No. 1, pp. 12-49, 1988.

[27] T.F. Cootes and C.J. Taylor, Statistical Models of Appearance for Computer Vision, Tech. Rep., University of Manchester, UK, 2004.

[28] B. van Ginneken, A.F. Frangi, J.J. Staal, B.M. ter Haar Romeny, and M.A. Viergever, "Active Shape Model Segmentation with Optimal Features," IEEE Trans. on Med. Imaging, Vol. 21, No. 8, pp. 924-933, 2002.

[29] M. Benjelloun, S. Mahmoudi, and F. Lecron, "A Framework of Vertebra Segmentation using the Active Shape Model-based Approach," Int. J. of Biomed. Imaging, Vol. 2011, pp. 114, 2011. 


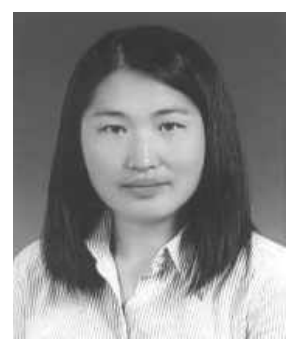

\section{Enkhbolor Adiya}

received the B.S., M.S. degrees in Applied Mathematics from School of Mathematics and Computer Science of National University of Mongolia in 2004, 2006 respectively. She is currently a $\mathrm{PhD}$ student in Medical Image Technology Laboratory (MITL), School of Computer Engineering, Inje University, South Korea. Her research interests include image enhancement and image segmentation.

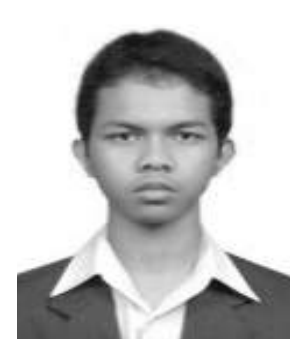

\section{Yonny Septian Izmantoko}

received the B.S. degree in Electrical Engineering from Institut Teknologi Bandung, Indonesia, in 2011. Currently, he is a master student in Computer Engineering department of Inje University, Gimhae, South Korea, joining Medical Image Technology Laboratory (MITL). His research interests are image segmentation, image visualization, and parallel data computing using GPU.

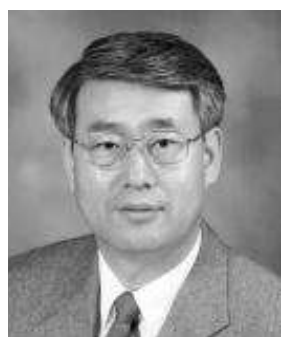

\section{Heung Kook Choi}

has gone the undergraduate studying and graduate studying in computer science and engineering at the Department of Electrical Engineering of Linköping University, Sweden (19841990) and Ph.D. studying in computerized image analysis at the Center for Image Analysis of Uppsala University, Sweden (1990-1996). He was President of Industry and Academic Cooperation Foundation at Inje University and now he is President of Korea Multimedia Society. His interesting research fields are in computer graphics, virtual reality, and medical image processing and analysis. 